\title{
Boston Bowel Preparation Scale
}

National Cancer Institute

\section{Source}

National Cancer Institute. Boston Bowel Preparation Scale. NCI Thesaurus. Code C138206.

A tool for reporting the quality of bowel preparation that was developed to limit interobserver variability, while preserving the ability to distinguish various degrees of bowel cleanliness. The the three broad regions of the colon: the right colon, the transverse colon, and the left colon are rated 0-3 and these segment scores are summed for a total BBPS score ranging from 0 to 9. 